\title{
Patterns of Mitochondrial DNA Damage in Blood and Brain Tissues of a Transgenic Mouse Model of Machado-Joseph Disease
}

\author{
Nadiya Kazachkova a, b Mafalda Raposo ${ }^{a}$ Rafael Montiel ${ }^{e}$ Teresa Cymbron a, b \\ Conceição Bettencourt ${ }^{a, b, f}$ Anabela Silva-Fernandesc, d Sara Silvac, d \\ Patrícia Maciel ${ }^{c, d}$ Manuela Lima ${ }^{a, b}$ \\ ${ }^{a}$ Center of Research in Natural Resources (CIRN), University of the Azores, Ponta Delgada, ${ }^{\mathrm{b}}$ Institute for \\ Molecular and Cell Biology (IBMC), University of Porto, Porto, 'Life and Health Sciences Research Institute (ICVS), \\ School of Health Sciences, University of Minho, Braga, and 'ICVS/3B's, PT Government Associate Laboratory, \\ Braga/Guimarães, Portugal; ' Laboratorio Nacional de Genómica para la Biodiversidad (LANGEBIO), CINVESTAV-IPN, \\ Irapuato, Mexico; ' Laboratorio de Biología Molecular, Instituto de Enfermedades Neurológicas, Fundación \\ Socio-Sanitaria de Castilla-La Mancha, Guadalajara, Spain
}

\section{Key Words}

Mitochondrial DNA • Mitochondrial DNA copy number and deletion $\cdot 3,867$-bp deletion $\cdot$ Machado-Joseph disease $\cdot$

SCA3 $\cdot$ CAG repeats $\cdot$ Ataxin- $3 \cdot$ Neurodegenerative disorder $\cdot$ Polyglutamine disorder $\cdot$ Transgenic mouse model

\begin{abstract}
Background: Machado-Joseph disease (MJD) is an autosomal dominant spinocerebellar ataxia caused by a CAG tract expansions in the ATXN3 gene. Patterns of mitochondrial damage associated with pathological findings of brain tissues could provide molecular biomarkers of this disorder. Objective: The potential of mitochondrial DNA (mtDNA) damage as a biomarker of MJD progression was investigated using a transgenic mouse model. Methods: DNA was obtained from affected (pontine nuclei) and nonaffected tissues (hippocampus and blood) of transgenic animals of three distinct age groups: 8 weeks, before onset of the phenotype; 16 weeks, at onset, and 24 weeks, at well-established phenotype. Wild-type littermate mice, serving as controls, were analyzed for the same tissues and age groups. mtDNA
\end{abstract}

damage was studied by fluorescence-based quantitative PCR in 84 transgenic and 93 wild-type samples. Results: A clear pattern of decrease in mtDNA copy number with age and accumulation of 3,867-bp deletions at the initial stages (both being more pronounced in transgenic mice) was observed. Pontine nuclei, the affected tissue in transgenic mice, displayed 1.5 times less copies of mtDNA than nonaffected brain tissue hippocampus (odds ratio $=1.21$ ). Pontine nuclei displayed the highest percentage of $\mathrm{mtDNA}$ deletions $(6.05 \%$ more in transgenic mice). Conclusion: These results suggest that mtDNA damage is related to the initiation of the phenotype in transgenic mice; mtDNA 3,867-bp deletions may be a biomarker of the initial stages of the disease.

Copyright $\odot 2012$ S. Karger AG, Basel

\section{Introduction}

Machado-Joseph disease (MJD/SCA3; MIM 109150) is a late-onset progressive neurological disorder, considered the most common autosomal dominant spinocerebellar ataxia worldwide $[1,2]$, reaching its highest prevalence in the Azores Islands (Portugal) (1/239 in the island of

\section{KARGER}

Fax +4161306 1234

E-Mail karger@karger.ch

www.karger.com
(C) 2012 S. Karger AG, Basel

$1660-2854 / 13 / 0114-0206 \$ 38.00 / 0$

Accessible online at:

www.karger.com/ndd
Nadiya Kazachkova

Department of Biology/CIRN, University of the Azores

Rua Mãe de Deus, Apartado 1422

PT-9501-801 Ponta Delgada, Azores (Portugal)

Tel. +351 296651 476, E-Mail nadya@uac.pt 
Flores, according to Bettencourt et al. [3]). Most MJD patients show the first symptoms in adulthood (mean age about 40 years), but onset extremes at 4 and 70 years have been described $[4,5]$. The MJD phenotype is complex and pleomorphic, involving the cerebellar, ocular motor, pyramidal, extrapyramidal and peripheral motor systems to variable degrees [5].

The ATXN3 gene (MIM 607047), the causative gene of MJD, spans a genomic region of $48 \mathrm{~kb}$, and contains a (CAG)n repeat tract encoding for glutamine repeats at exon $10[6,7]$. Wild-type alleles have 12-44 CAG repeats while, consensually, the mutant alleles contain from 61 to $87 \mathrm{CAG}$ repeats [8]. The size of the (CAG)n tract in the expanded alleles negatively correlates with the age at onset [9]. The ATXN3 gene encodes for ataxin-3, which, in its mutated form, presents an elongation of a polyglutamine (poly-Q) tract near its C-terminus [10]. Despite the ubiquitous expression of ataxin-3 in brain and nonbrain tissues, selective neuronal cell death has been reported [e.g. ref. 11, 12]. Neuropathological studies typically reveal neuronal loss in the cerebellar dentate nucleus, pons, substantia nigra, thalamus, globus pallidus, anterior horn cells and Clarke's column in the spinal cord, vestibular nucleus, many cranial motor nuclei as well as other brainstem nuclei [12]. The mechanisms by which mutant ataxin-3 induces neurodegeneration have been extensively studied but remain incompletely understood [reviewed in ref. 13]. Difficulties in fully understanding the pathogenic process leading to the neurotoxicity of mutant ataxin-3 are reflected in the current lack of specific strategies to reverse morbidity. Several clinical trials using drugs that aim to delay disease progression are ongoing. A crucial aspect, which may compromise the success of clinical trials in MJD, is the lack of measurement tools to monitor disease progression and detect subtle therapeutic benefits. Molecular biomarkers of progression are therefore urgently needed in this disorder.

Morphological, biochemical, genetic as well as cell and animal model studies reveal that mitochondrial damage is an important factor in aging and age-related neurodegeneration [14]. Mitochondria are known to play a pivotal role in many metabolic and apoptotic pathways that regulate cellular life and death. Mitochondrial DNA (mtDNA) alterations, including quantitative and qualitative changes, have been reported in several neurodegenerative disorders. In poly-Q disorders, namely in Huntington's disease, the accumulation of large deletions in mtDNA has been described [15]. Although mtDNA damage in MJD has already been reported in the literature [16, 17], including depletion and deletion, in peripheral cells of patients and in cellular models of the disease, no investigations on the association between neuropathological findings and patterns of mtDNA damage have been performed so far due to difficulties in obtaining brain tissues. To overcome those difficulties, we used a transgenic mouse model of MJD (CMVMJD94) in the present study, which expresses the full-length human ataxin-3 and displays a motor phenotype in the absence of evident cell death [18]. The main goal was to investigate the relationship between damage to mtDNA (copy number depletion, as well as the accumulation of deletions), and the initiation and progression of the transgenic mouse phenotype. Our aims were: (a) to identify a pattern of mtDNA damage in affected brain tissue as well as nonaffected (brain and nonbrain) tissues associated with the development of the phenotype; (b) to define the effect of aging on the accumulation of mtDNA damage, and its tissue specificity. To fulfill these objectives, mtDNA copy number in distinct tissues of the transgenic mice (brain and nonbrain/affected and nonaffected) and at different stages relatively to the onset of the phenotype, was determined and compared with values obtained in controls. The presence of the mtDNA 3,867-bp deletion, the homolog of the human mtDNA 4977-bp deletion, considered as a marker of aging, was also quantified and compared with values obtained in controls.

\section{Materials and Methods}

\section{Mouse Model and Experimental Design}

A novel cDNA transgenic mouse model of the early stages of MJD (Mus musculus, strain C57B1/6, line CMVMJD94) developed by P. Maciel's group was used in the present study [18]. These transgenic mice ubiquitously express the full-length mutant human ataxin-3, under the regulation of the cytomegalovirus promoter, and display a motor phenotype. The mouse model used also mimics some key features that are common in MJD patients, namely CAG repeat instability, neurological damage and brain pathology. Therefore, it has been proposed as a useful model for dissecting the initial cellular and molecular events in the pathogenesis of MJD [18]. DNA from 28 transgenic mice carrying the expanded repeat (98-104 CAGs) (cases) was compared with DNA from 31 wild-type littermate mice (C57Bl/6 strain), matched by sex and age (controls). Unaffected brain tissue (hippocampus) and affected brain tissue (pontine nuclei), as well as a nonbrain tissue (blood) were collected from transgenic mice. These three tissues were obtained from each animal and were analyzed within three age groups defined as follows: 8-week-old, corresponding to the age prior to the appearance of the phenotype; 16-week-old, corresponding to the age at onset; 24 -week-old, corresponding to the age at which the phenotype is well established. The same tissues of mice of the same age groups were studied in control animals (8-11 animals analyzed per group). 
The animals were maintained in accordance with European regulations (European Union Directive 86/609/EEC). Animal facilities and the people directly involved in animal experiments were certified by the Portuguese regulatory entity - Directorate General for Veterinary Medicine. All protocols were approved by the joint Animal Ethics Committee of the Life and Health Sciences Research Institute, University of Minho, and the Institute for Molecular and Cell Biology, University of Porto, Porto, Portugal.

DNA Isolation and Molecular Analysis of the (CAG)n Repeats

DNA was extracted from tail biopsies (blood) or from macrodissected brain tissues (hippocampus and pontine nuclei) using the Puregene DNA isolation kit (Gentra Systems, Minneapolis, Minn., USA), and the size of the CAG tract was assessed as previously described [18].

\section{Quantitative Real-Time PCR}

Determination of the mtDNA copy number and quantitative detection of the mtDNA 3867-bp deletion were performed by fluorescence-based quantitative real-time PCR using the Applied Biosystems 7900HT real-time PCR System (Applied Biosystems, Foster City, Calif., USA). mtDNA quantity was corrected by simultaneous measurement of a nuclear Gapdh gene (TaqMan Rodent Gapdh Control, P/N 4308313, Applied Biosystems; the nDNA-specific fluorescent probe was labeled internally using VIC fluorescent dye) by the 'absolute quantitation' (standard curve) method. Primers and TaqMan MGB probes (minor groove binder, labeled at the $5^{\prime}$-end with a fluorescent reporter, 6-FAM) were designed for the sequence outside the deletion-specific region (mtDNA ${ }^{\mathrm{ALL}}$; mtDNA copy number measurement) and inside the deletion-specific region ( $m$ tDNA ${ }^{\text {NORMAL}}$; detection of the mtDNA 3,867-bp deletion) using Primer Express v3.0 (Applied Biosystems). The primers used were: $m \operatorname{mDNA}^{\mathrm{ALL}}\left(5^{\prime}-3^{\prime}\right)$ forward - TCGCCACTTCTATAACAGCTATG, reverse - AATGCTAGGCGTTTGATTGG, probe mtDNA ${ }^{\text {ALL }}$ (6-FAM MGBNFQ) GCAGACGAACAAGACATC; primers mtDNA NORMAL $\left(5^{\prime}-3^{\prime}\right)$ forward - TGTACCCACGCATTCTTCAA, reverse - AATGCTAGGCGTTTGATTGG, probe mtDNA ${ }^{\text {NORMAL }}$ (6-FAM MGBNFQ) ACGAAAATGACCCAGACCTC. Primer sequences were screened by BLASTn to eliminate any homology to human DNA and mouse nuclear DNA and were also screened against the NCBI SNP database. A total volume of $20 \mu \mathrm{l}$ was used in PCRs containing: $20 \mathrm{ng}$ of template DNA, 5 pmol of each primer and probe, $10 \times$ buffer, 1.5 units of BioTaq DNA polymerase (Bioline), $1.25 \mathrm{mM} \mathrm{MgCl}_{2}, 0.2 \mathrm{mM}$ dNTPs and $1 \mu \mathrm{l}$ of TaqMan Rodent Gap$d h$ Control. The thermal cycling conditions were as follows: $95^{\circ} \mathrm{C}$ for $10 \mathrm{~min}$, followed by 40 cycles of $95^{\circ} \mathrm{C}$ for $15 \mathrm{~s}$, and $59^{\circ} \mathrm{C}$ for $1 \mathrm{~min}$. Every sample was tested in triplicate. In each PCR run, serial dilutions of the mouse genomic DNA with known amounts of input nuclear copy number were included to create a standard reference curve. The absolute mtDNA copy number (mtDNA ${ }^{\mathrm{ALL}}$ ) was calculated as described in the manual Creating Standard Curves with Genomic DNA or Plasmid DNA Templates for Use in Quantitative PCR by Applied Biosystems (www6.appliedbiosystems.com/support/tutorials/pdf/quant_pcr.pdf). The number of mtDNA copies with deletions was calculated by the difference of total mtDNA copies (mtDNA ${ }^{\mathrm{ALL}}$ ) and mtDNA copies without deletions (mtDNA $\left.{ }^{\mathrm{NORMAL}}\right)$.

\section{Statistical Analysis}

Copy Number Macro (Applied Biosystems) and SDS software, version 2.X, were used to process the data. The amount of mtDNA per cell was calculated and the results are expressed as mean copy number for three fluorescence-based quantitative real-time PCRs. Statistical analysis was performed using Statistica software package, version 7.0 [19]. A p value less than 0.05 was considered statistically significant. Odds ratios (ORs) were calculated from the data presented in figure 1 . Statistical association between variables was assessed by multiple regression analysis. Total mtDNA copy number and percentage of mtDNA copies with deletions were used as dependent variables. Age, mtDNA origin (tissue), sample origin (wild type or transgenic) and number of CAG repeats were used as predictors in multiple regression analysis. Multiple regression analysis was performed for: (a) all samples with sample origin (wild type or transgenic) and number of CAG repeats as predictors; (b) transgenic samples with age, mtDNA origin (tissue) and number of CAG repeats as predictors, and (c) wild-type samples with age and tissue as predictors. In multiple regression analysis, $\beta$-coefficients were calculated to measure the individual contribution of each predictor. The significance of the contribution of a predictor is described by the $t$ and $\mathrm{p}$ values (the smaller the $\mathrm{p}$ value and the larger the $\mathrm{t}$ value, the greater the contribution of a particular predictor). The cumulative contribution of predictors is described by multiple correlation coefficients ( $r$ ). $r^{2}$ values show how much of the variability in the outcome is accounted for by the predictors. Adjusted $r^{2}$ values give an idea how the model generalizes, the closer the values, the better the model. The F ratio from the ANOVA analysis represents the ratio of the improvement in prediction that results from fitting the model relative to the inaccuracy that still exists in the model ('residual').

The mean values of one group of variables were compared with the mean of another group using Fisher's least significant difference test.

\section{Results}

\section{Findings in Wild-Type Animals}

Results revealed that the total mtDNA copy number of older mice was reduced when compared with that of younger mice, thus indicating the effect of aging on the accumulation of mtDNA depletions ( $\mathrm{p}<0.001$, table 1 , model B). A 1.59-fold decrease in total mtDNA copy number from week 8 to week 24 was observed. The mean percentage of mtDNA copies with deletions reached its maximum in 8 -week-old mice (up to $58 \%$ in pontine nuclei) (fig $1,2 \mathrm{c}$ ), again decreasing with age (table 1).

Among brain tissues, pontine nuclei had a lower mean mtDNA copy number (fig. 2b). Additionally, the highest mean level of mtDNA deletions was also observed in pontine nuclei (fig. 1, 2d). 


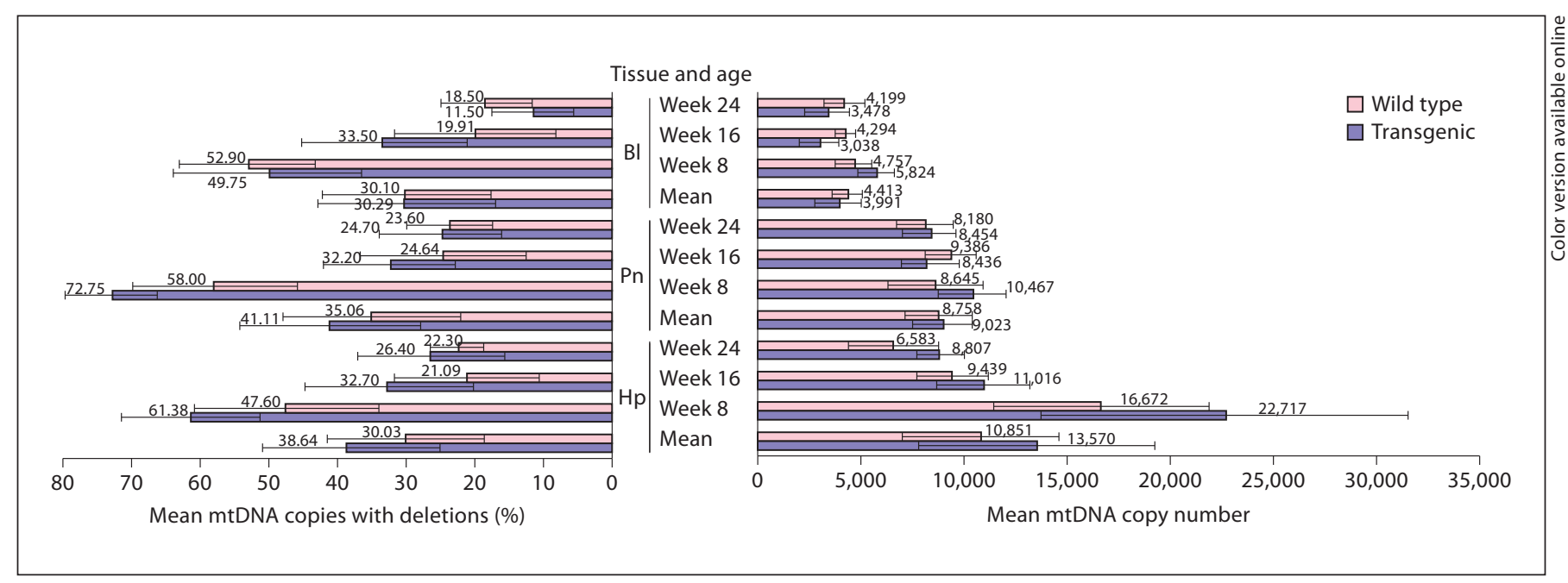

Fig. 1. Mean mtDNA copy number and percentage of mtDNA copies with deletions in different tissues and ages of transgenic and wild-type mice. $\mathrm{Hp}=$ Hippocampus; $\mathrm{Pn}=$ pontine nuclei; $\mathrm{Bl}=$ blood; bars on the diagram represent standard deviation; numbers above/at the bars represent actual value of mean mtDNA copy number or percentage of mtDNA copies with deletions; mean value for each tissue represents the mean value for weeks 8,16 and 24 .

Table 1. Parameters estimates of multiple regression models

\begin{tabular}{|c|c|c|c|c|c|c|c|c|}
\hline & \multicolumn{4}{|c|}{ mtDNA copy number } & \multicolumn{4}{|c|}{ mtDNA copies with deletions, $\%$} \\
\hline & $\beta$ & $95 \% \mathrm{CI}$ & $\mathrm{p}$ value & t value & $\beta$ & $95 \%$ CI & $\mathrm{p}$ value & $\mathrm{t}$ value \\
\hline Sample origin ${ }^{1}$ & -0.75 & -6.82 to 5.31 & 0.81 & -0.25 & -9.25 & -15.14 to -3.36 & $<0.001$ & -3.10 \\
\hline$(\mathrm{CAG}) \mathrm{n}$ & -0.69 & -6.75 to 5.37 & 0.82 & -0.23 & -9.16 & -15.05 to -3.27 & $<0.001$ & -3.07 \\
\hline \multicolumn{9}{|l|}{ Model B } \\
\hline$(\mathrm{CAG}) \mathrm{n}$ & -0.74 & -7.44 to 5.96 & 0.83 & -0.22 & -10.80 & -16.67 to -4.93 & $<0.001$ & -3.66 \\
\hline \multicolumn{9}{|l|}{ Model C } \\
\hline Age & -0.15 & -0.31 to 0.00 & 0.047 & -2.01 & -0.58 & -0.75 to -0.41 & $<0.001$ & -6.88 \\
\hline Tissue & -0.66 & -0.81 to -0.51 & $<0.001$ & -8.60 & -0.11 & -0.28 to 0.06 & 0.20 & -1.28 \\
\hline
\end{tabular}

Multiple regression analysis was performed to find out how mtDNA copy number and percentage of mtDNA copies with deletions (used as dependent variables) of the samples analyzed are affected by other variables (predictors), such as age, mtDNA origin (tissue) and (CAG)n. Three models were analyzed, which differed in predictors and sample origin (transgenic or wild type).

Model A: mtDNA copy number and percentage of mtDNA copies with deletions (dependent variables) versus sample origin (transgenic or wild type) and (CAG)n (predictors), all samples.
Model B: mtDNA copy number and percentage of mtDNA copies with deletions (dependent variables) versus age, mtDNA origin (tissue) and (CAG)n (predictors), transgenic samples.

Model C: mtDNA copy number and percentage of mtDNA copies with deletions (dependent variables) versus age and mtDNA origin (tissue) (predictors), wild-type samples.

${ }^{1}$ Transgenic or wild type; $\beta=\beta$-coefficient; $95 \% \mathrm{CI}=$ confidence interval at $95 \%$ confidence level. 


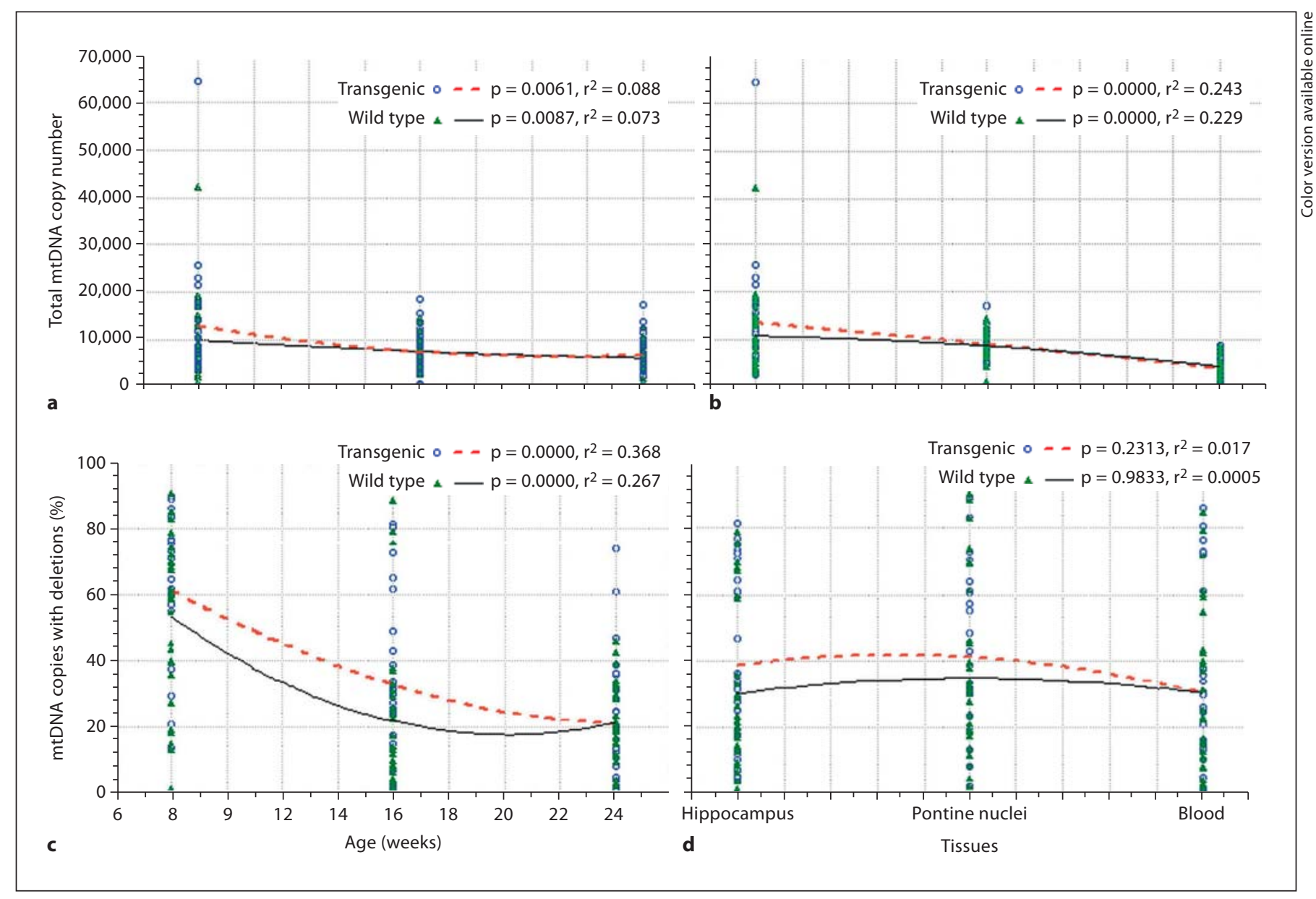

Fig. 2. Correlation of mtDNA copy number and percentage of mtDNA copies with deletions with age and mtDNA origin (tissue). a mtDNA copy number versus age. b mtDNA copy number versus tissue. c Percentage of mtDNA copies with deletions versus age. $\mathbf{d}$ Percentage of mtDNA copies with deletions versus tissue.

\section{Wild-Type versus Transgenic Animals}

Decrease in mtDNA Copy Number with Age

Compared to wild-type mice, transgenic animals demonstrated more evident accumulation of mtDNA depletion with age $\left(r^{2}=0.088, p=0.006\right.$ vs. $r^{2}=0.073, p=$ 0.009 in wild-type mice, fig. $1,2 \mathrm{a}$, online suppl. table 1 ; see www.karger.com/doi/10.1159/000339207 for all online suppl. material). The mean decrease in mtDNA copy number with age in all tissues was 1.22 times higher in transgenic animals (OR for hippocampus $=1.02$, OR for pontine nuclei $=1.17$, and OR for blood $=1.48$ ). Comparison of mtDNA copy number in all tissues of transgenic mice between the initial stage of the disease (presymptomatic versus onset, 8-16 weeks) and the later stage of the disease (onset versus well-established phenotype, 16-
24 weeks) showed a higher difference for the initial stage of the disease ( $\mathrm{OR}=1.24-2.21)$. While in wild-type mice, a decrease in mtDNA copy number between $8-16$ versus 16-24 weeks was observed only in blood and the hippocampus $(\mathrm{OR}=1.09$ and 1.24 , respectively; $\mathrm{OR}=0.92$ for blood), but, once again, this difference was more evident in transgenic mice ( $\mathrm{OR}=1.33$ for the hippocampus, and $\mathrm{OR}=2.03$ for blood). Concerning the pontine nuclei, this difference was also more evident in transgenic mice $(\mathrm{OR}=1.55)$.

Accumulation of mtDNA Deletions at the Initial Stage of the Disease

Compared to wild-type mice, transgenic animals demonstrated more evident accumulation of deletions (up to $72.75 \%$ in the pontine nuclei of transgenic vs. $58 \%$ of wild- 
type animals) (fig. $1 ; \mathrm{p}<0.001, \mathrm{r}^{2}=0.37$ and 0.27 for transgenic and wild type, respectively; fig. 2c). Samples of almost all tissues of transgenic mice had a much higher mean percentage of mtDNA copies with deletions compared to wild-type mice. The difference in mean percentage of mtDNA copies with deletions between transgenic and wild-type mice ranged from $1.1 \%$ (pontine nuclei, 24 weeks) to $14.75 \%$ (pontine nuclei, 8 weeks) (calculated from fig. 1). Multiple regression analysis demonstrated that the percentage of mtDNA deletions is significantly influenced by sample origin (wild type or transgenic) (table 1, model A, $\mathrm{p}<0.001$ ). Brain tissues of transgenic mice and all tissues of wild-type mice showed statistically significant differences between mean deletion values for 8 versus 16 weeks (corresponding to presymptomatic stage and onset in transgenic mice) as well as for 8 versus 24 weeks (corresponding to presymptomatic stage and well-established phenotype in transgenic mice). For blood, a statistically significant difference in transgenic animals was found between the presymptomatic stage and well-established phenotype (8 vs. 24 weeks), as well as between onset and wellestablished phenotype (16 vs. 24 weeks). On the other hand, when comparing transgenic with wild-type mice of the same age, significant differences between mean deletion values were observed for all tissues for 8 versus 16 weeks and 8 versus 24 weeks, with more pronounced changes in cells of transgenic mice (online suppl. table 2).

\section{More Evident Decrease in mtDNA Copy Number}

with Age and Highest Accumulation of mtDNA

Deletions in Pontine Nuclei

The decrease in mtDNA copy number with age among brain tissues was more pronounced in pontine nuclei (the affected tissue in transgenic mice) (OR for the hippocampus $=1.02$, OR for pontine nuclei $=1.17$ ). Pontine nuclei had a lower global mean mtDNA copy number compared with the hippocampus in both transgenic and wild-type mice (1.5 and 1.24 times less copies, respectively, OR = 1.21). Mean accumulation of mtDNA deletions was also the highest in pontine nuclei $(6.05 \%$ more in transgenic than in wild-type mice; $2.47 \%$ more than in the hippocampus and $10.82 \%$ more than in blood for transgenic mice; fig. 1). Both results demonstrate that thte pontine nuclei were likely to have more mtDNA damage than the hippocampus and blood (affected vs. nonaffected tissues in transgenic mice).

\section{Findings in Blood}

In order to evaluate the application potential of the results obtained using brain tissues in the mouse model, in the context of biomarker identification, special attention was paid to mtDNA damage in blood, a biological material whose collection is minimally invasive, rapid and appropriate for multiple sampling. Findings of mtDNA damage in blood of transgenic animals were similar to those in affected tissue of pontine nuclei, namely a decrease in mtDNA copy number from week 8 to week 16 and almost no changes or a slight increase from week 16 to week 24, the highest percentage of mtDNA copies with deletions being observed in week 8 , with a subsequent decrease (fig. 1). Although, in contrast to other tissues, blood demonstrated higher percentages of mtDNA copies with deletions in wild-type mice compared with transgenic mice at 24 weeks, it followed a pattern of a prominent decrease in the percentage of mtDNA copies with deletions from week 16 to week 24 in transgenic mice compared with a slight decrease in wild-type mice (22 vs. $1.41 \%$, OR $=15.6$ ), which is characteristic for brain tissues. And, importantly, at week 16 , which corresponds to the onset in transgenic mice, the percentage of mtDNA copies with deletions in blood was much higher in transgenic mice (13.59\%).

\section{Global Analysis}

A large part of the variation in mtDNA copy number and percentage of mtDNA copies with deletions was accounted for by age, mtDNA origin (tissue) and number of CAG repeats (for transgenic mice). In transgenic mice, these variables had a higher impact on the percentage of mtDNA copies with deletions ( $40.7 \%$ of the variation, $\mathrm{p}<$ 0.001 . Table 2 , model B) while in wild-type mice, the impact was higher on total mtDNA copy number $(47.3 \%$ of the variation, $\mathrm{p}<0.001$; table 1,2 , model $\mathrm{C}$ ).

The above-mentioned results indicate a clear difference between transgenic and wild-type mice, which is expressed as more evident accumulation of mtDNA depletion with age, starting from week 8 (OR up to 1.48 for transgenic vs. wild-type mice), and more evident accumulation of mtDNA deletions (up to 72.75 vs. $58 \%$ of wild-type animals) in most tissues of transgenic animals. Several key traits in mtDNA dynamics can be highlighted: decrease in mtDNA copy number with age, accumulation of deletions at the initial stages (both more pronounced in transgenic mice). A more evident decrease in mtDNA copy number with age (comparing brain tissues), and the highest accumulation of mtDNA deletions (comparing all tissues) was observed in the affected tissue. Most tissues demonstrated a decline in mtDNA copy number (except pontine nuclei and blood in 16- to 24 -week-old transgenic mice, and pontine nuclei in 8 - to 
Table 2. Summary of multiple regression models (mtDNA copy number and percentage of mtDNA copies with deletions versus age, mtDNA origin (tissue) and (CAG)n)

\begin{tabular}{|c|c|c|c|c|c|}
\hline & Multiple $r$ & Multiple $r^{2}$ & Adjusted $\mathrm{r}^{2}$ & F value & $\mathrm{p}$ value \\
\hline \multicolumn{6}{|l|}{ Model A } \\
\hline mtDNA copy number & 0.065 & 0.004 & -0.007 & 0.372 & 0.690 \\
\hline \multicolumn{6}{|l|}{ Model B } \\
\hline mtDNA copy number & 0.476 & 0.226 & 0.187 & 5.777 & $<0.001$ \\
\hline mtDNA copies with deletions, $\%$ & 0.638 & 0.407 & 0.377 & 13.546 & $<0.001$ \\
\hline mtDNA copy number & 0.688 & 0.473 & 0.462 & 40.469 & $<0.001$ \\
\hline mtDNA copies with deletions, $\%$ & 0.600 & 0.360 & 0.345 & 25.263 & $<0.001$ \\
\hline
\end{tabular}

Model A: mtDNA copy number and percentage of mtDNA copies with deletions (dependent variables) versus sample origin (transgenic or wild type) and (CAG)n (predictors), all samples.

Model B: mtDNA copy number and percentage of mtDNA copies with deletions (dependent variables) versus age, mtDNA origin (tissue) and (CAG)n (predictors), transgenic samples.
Model C: mtDNA copy number and percentage of mtDNA copies with deletions (dependent variables) versus age and mtDNA origin (tissue) (predictors), wild-type samples.

$\mathrm{r}=$ Multiple correlation coefficient; $\mathrm{r}^{2}=$ squared multiple correlation coefficient. 16-week-old wild-type mice) and an unexpected decline in the percentage of mtDNA copies with deletions (except for the hippocampus in 8- to 16-week-old wild-type mice) with age (fig. 1, 2). Blood showed the lowest values for both mtDNA copy number and percentage of mtDNA copies with deletions, but demonstrated a pattern of mtDNA damage similar to that of pontine nuclei in transgenic animals. The affected tissue had lower mtDNA copy numbers in comparison with nonaffected brain tissue, and the highest percentage of mtDNA copies with deletions among all tissues (fig. 1).

\section{Discussion}

Previous studies have shown that mtDNA alterations could play a role in the pathogenesis of poly- $\mathrm{Q}$ diseases $[14,16,17,20,21]$. For MJD, the evidence concerning mtDNA alterations is scarce. Liu et al. [16], for instance, demonstrated that leukocytes of patients present significant mtDNA depletions compared with healthy controls. These authors, however, did not take into account the age at disease onset and the time of disease progression: this severely limits the analysis since, for example, they were not able to take into account the time elapsed since onset, which is crucial, considering that disease-associated mtDNA damage may depend on disease progression itself. Moreover, no investigations on the association between neuropathological findings and patterns of dam- age have been performed so far, due to difficulties in obtaining brain tissues. Using stably transfected cells with 78 CAGs, Yu et al. [17] demonstrated that the mtDNA copy number in such cells was lower than that of wildtype lines. This trend for depletion was also observed for mtDNA obtained from leukocytes of MJD patients; furthermore the frequency of the 4977-bp deletion was higher than in the matching healthy controls, an indication of higher damage in the mtDNA molecules of patients. Again, in this work, the lack of phenotypic data and the reduced number of cases precludes any conclusions as to the interplay between mtDNA damage and the evolution of the disease. Although not conclusive, due to the limitations referred to, previous research conducted on mtDNA damage and MJD led us to consider the possibility that there is a pattern of mitochondrial alterations associated with disease progression. Thus, the novelty of our study resided in the use of a mouse model and in comparing distinct tissues (affected and nonaffected) and different stages relatively to the onset of the phenotype, allowing us to draw some conclusions concerning the relationship between the evolution of the disease and the pattern of damage in affected and nonaffected tissues.

In this study, we analyzed in vivo how changes in mtDNA copy number and the accumulation of mtDNA deletions were related to the MJD transgenic mouse phenotype and its progression at the early stages of the disease. A more pronounced decrease in mtDNA copy number with age and accumulation of deletions at the initial 
stage of the disease were observed in transgenic mice. Among brain tissues, a more evident decrease in mtDNA copy number with age was observed in pontine nuclei, the affected brain region. Accumulation of mtDNA deletions was, furthermore, also highest in the affected tissue. Consistent with the findings of previous research [16, 17], aging was shown to be associated with mtDNA depletion; within brain tissues, this depletion was more pronounced in the affected tissue (pontine nuclei) of transgenic mice compared to wild-type animals. Although not reaching statistical significance for some groups, age and tissue were found to have a significant impact on mtDNA copy number, as evidenced by multiple regression analysis. The lack of significance in some of the groups may be due to the small sample size $(n=8-11)$ or quite high level of the standard deviations in the groups. The difference in mtDNA copy number at the initial stages of the disease was found to be higher than at later stages, and it was up to twice as high in transgenic animals compared to wildtype animals of the same age groups. Thus, the progressive depletion associated with aging is more evident in transgenic mice. This pattern was more evident in pontine nuclei (when comparing brain tissues). Globally, the results obtained indicate that mtDNA damage could be related to the initiation of the phenotype in this MJD transgenic mouse model.

The mtDNA 3,867-bp deletion causes removal or truncation of six structural (ATPase 6/8, COIII, ND3, ND4L, and ND4) and five tRNA genes. In contrast to previous observations $[15,17]$, which are consistent with expectations of higher rates of mtDNA deletion at the late stages of the disease, we observed that the mtDNA 3,867-bp deletion was more likely to be present in younger transgenic mice, in accordance with the recent results of Chen et al. [22]. However, compared to wild-type mice, transgenic mice in general demonstrated higher percentages of mtDNA copies with deletions, supporting the previous findings in healthy and affected MJD individuals [17]. A possible explanation for the accumulation of mtDNA deletions at earlier ages could be that the cells, which are still alive at later ages, are the ones without the high percentage of deletion, while those with the deletion die, resulting in a lower percentage of deletions at later ages. Among the tissues analyzed, the highest numbers of mtDNA deletions were observed in the affected tissue, the pontine nuclei. We speculate that the pontine nuclei may be particularly sensitive to damage and that the MJD-causing mutation affects pontine nuclei cells by changing their stress response. As a result, the antioxidant activity of cells is reduced, leading to an

increase in the generation of reactive oxygen species and the ensuing mitochondrial damage, which in turn results in stress-induced apoptosis during the progression of MJD.

Our results seem to support a possible higher oxidative injury in cells with mutant ATXN3 because a decrease in mtDNA copy number with age and accumulation of deletions (especially at the initial stages) were more evident in transgenic mice. We can thus hypothesize that the relative number of mtDNA 3,867-bp deletions is related to the stage of the disease.

Based on the findings made in this study, we can suggest that mtDNA damage has a potential to be used as a biomarker of phenotype initiation in MJD. Further studies using other models and a large number of patient samples are needed to confirm these findings, particularly for accessible tissues such as blood, and could represent a powerful tool in the context of drug trials.

\section{Acknowledgments}

This study was supported by the following grants: DRCT Postdoctoral fellowship to N.K. (M3.1.7/F/002/2008), FCT Postdoctoral fellowship to T.C. (SFRH/BPD/38659/2007) and C.B. (SFRH/BPD/63121/2009), FCT research grants to S.S. (PTDC/ SAU-GMG/64076/2006) and A.S.F. (PIC/IC/83013/2007).

\section{Disclosure Statement}

The authors declare that they have no conflict of interest.

References

Neurodegener Dis 2013;11:206-214
Bettencourt C, Lima M: Machado-Joseph disease: from first descriptions to new perspectives. Orphanet J Rare Dis 2011;6:35.

- 2 Schols L, Bauer P, Schmidt T, Schulte T, Riess O: Autosomal dominant cerebellar ataxias: clinical features, genetics, and pathogenesis. Lancet Neurol 2004;3:291-304.

-3 Bettencourt C, Fialho RN, Santos C, Montiel R, Bruges-Armas J, Maciel P, Lima M: Segregation distortion of wild-type alleles at the Machado-Joseph disease locus: a study in normal families from the Azores islands (Portugal). J Hum Genet 2008;53:333-339.

-4 Carvalho DR, La Rocque-Ferreira A, Rizzo IM, Imamura EU, Speck-Martins CE: Homozygosity enhances severity in spinocerebellar ataxia type 3. Pediatr Neurol 2008;38: 296-299.

5 Coutinho P: Doença de Machado-Joseph: tentativa de definição; diss., Porto, 1992. 
6 Ichikawa Y, Goto J, Hattori M, Toyoda A, Ishii $\mathrm{K}$, Jeong SY, Hashida $\mathrm{H}$, Masuda $\mathrm{N}$, Ogata K, Kasai F, Hirai M, Maciel P, Rouleau GA, Sakaki Y, Kanazawa I: The genomic structure and expression of MJD, the Machado-Joseph disease gene. J Hum Genet 2001; 46:413-422.

7 Kawaguchi Y, Okamoto T, Taniwaki M, Aizawa M, Inoue M, Katayama S, Kawakami H, Nakamura S, Nishimura M, Akiguchi I, Kimura J, Narumiya S, Kakizuka A: CAG expansions in a novel gene for Machado-Joseph disease at chromosome 14q32.1. Nature Genet 1994;8:221-228.

8 Maciel P, Costa MC, Ferro A, Rousseau M, Santos CS, Gaspar C, Barros J, Rouleau GA, Coutinho P, Sequeiros J: Improvement in the molecular diagnosis of Machado-Joseph disease. Arch Neurol 2001;58:1821-1827.

-9 Maciel P, Gaspar C, DeStefano AL, Silveira I, Coutinho P, Radvany J, Dawson DM, Sudarsky L, Guimarães J, Loureiro JE, Nezarati MM, Corwin LI, Lopes-Cendes I, Rooke K, Rosenberg R, MacLeod P, Farrer LA, Sequeiros J, Rouleau GA: Correlation between CAG repeat length and clinical features in Machado-Joseph disease. Am J Hum Genet 1995;57:54-61.
10 Orr HT, Zoghbi HY: Trinucleotide repeat disorders. Annu Rev Neurosci 2007;30:575621.

11 Paulson HL, Perez MK, Trottier Y, Trojanowski JQ, Subramony SH, Das SS, Vig P, Mandel JL, Fischbeck KH, Pittman RN: Intranuclear inclusions of expanded polyglutamine protein in spinocerebellar ataxia type 3. Neuron 1997;19:333-344.

12 Yamada M, Hayashi S, Tsuji S, Takahashi H: Involvement of the cerebral cortex and autonomic ganglia in Machado-Joseph disease. Acta Neuropathol 2001;101:140-144.

13 Paulson H: Machado-Joseph disease/spinocerebellar ataxia type 3 . Handb Clin Neurol 2011;103:437-449.

14 Martin LJ: Mitochondrial and cell death mechanisms in neurodegenerative diseases. Pharmaceuticals (Basel) 2010;3:839-915.

15 Stack EC, Matson,WR, Ferrante RJ: Evidence of oxidant damage in Huntington's disease: translational strategies using antioxidants. Ann NY Acad Sci 2008;1147:79-92.

16 Liu C, Cheng W, Kuo S, Li JY, Soong BW, Wei YH: Depletion of mitochondrial DNA in leukocytes of patients with poly-Q diseases. J Neurol Sci 2008;264: 18-21.

17 Yu Y, Kuo C, Cheng W, Liu C, Hsieh M: Decreased antioxidant enzyme activity and increased mitochondrial DNA damage in cellular models of Machado-Joseph disease. J Neurosci Res 2009;87:1884-1891.
8 Silva-Fernandes A, Costa MdoC, DuarteSilva S, Oliveira P, Botelho CM, Martins L, Mariz JA, Ferreira T, Ribeiro F, CorreiaNeves M, Costa C, Maciel P: Motor uncoordination and neuropathology in a transgenic mouse model of Machado-Joseph disease lacking intranuclear inclusions and ataxin-3 cleavage products. Neurobiol Dis 2010;40: 163-176.

19 Hill T, Lewicki P: Statistics Methods and Applications: A Comprehensive Reference for Science, Industry, and Data Mining. Tulsa, StatSoft, 2006.

20 Lin MT, Beal MF: Mitochondrial damage and oxidative stress in neurodegenerative diseases. Nature 2006;443: 787-795.

21 Su S, Jou S, Cheng W, Lin T, Li J, Huang C, Lee Y, Soong B, Liu C: Mitochondrial DNA damage in spinal and bulbar muscular atrophy patients and carriers. Clin Chim Acta 2010;411: 626-630.

22 Chen T, He J, Shen L, Fang H, Nie H, Jin T, Wei X, Xin Y, Jiang Y, Li H, Chen G, Lu J, Ba Y: The mitochondrial DNA 4,977-bp deletion and its implication in copy number alteration in colorectal cancer. BMC Med Genet $2011 ; 13: 12-18$ 